\title{
Hallazgos de la Educación Inclusiva y la Pedagogía teatral: actualización curricular en un Centro de Atención Integral para Personas con Discapacidad en Costa Rica
}

Findings of Inclusive Education and Theater Pedagogy: curricular update for a Center of Comprehensive Care for People with Disabilities in Costa Rica

\section{Volumen 20, Número 2 \\ Mayo - Agosto \\ pp. 1-24}

Melissa Rodríguez Ríos

\section{Citar este documento según modelo APA}

Rodríguez Ríos, Melissa. (2020). Hallazgos de la Educación Inclusiva y la Pedagogía teatral: actualización curricular en un Centro de Atención Integral para Personas con Discapacidad en Costa Rica. Revista Actualidades Investigativas en Educación, 20(2), 1-24. Doi. 10.15517/aie.v20i2.41663 


\title{
Hallazgos de la Educación Inclusiva y la Pedagogía teatral: actualización curricular en un Centro de Atención Integral para Personas con Discapacidad en Costa Rica
}

Findings of Inclusive Education and Theater Pedagogy: curricular update for a Center of Comprehensive Care for People with Disabilities in Costa Rica

\section{Melissa Rodríguez Ríos ${ }^{1}$}

\begin{abstract}
Resumen: En el presente artículo se identifican las necesidades educacionales actuales, así como los retos y alcances pedagógico-administrativos que conllevan la actualización del plan de estudios ofrecido por la Asociación Pro Niño y Adulto Excepcional (APNAE) para que se integre la pedagogía teatral como materia regular. Por ello, se contextualiza el estado de la Educación Inclusiva y la Pedagogía Teatral en Costa Rica. Luego, se exponen los ejes conceptuales de la investigación, los cuales dan paso a las categorías de análisis que se explican por medio de la metodología cualitativa, la cual consistió en un diagnóstico participativo aplicado durante el 2018. La población participante se definió en tres grupos de estudio: el primer grupo conformado por cuatro docentes del APNAE, el segundo grupo por cuatro personas de núcleos familiares de estudiantes de la APNAE (dos de ellos pertenecientes a la Junta Directiva) $y$, por último, con doce personas con distintas condiciones de discapacidad (personas con Síndrome de Down, discapacidad intelectual leve y medio, y discapacidades motoras), los cuales se dividieron en dos grupos de trabajo. La limitación de esta indagación reside en que no se integró al cuerpo institucional (CONAPDIS, Departamento de Apoyos Educativos para el estudiantado con discapacidad del Ministerio de Educación Pública de Costa Rica, así como otras personas y entes claves del Ministerio de Cultura y Juventud) como parte de los sujetos de investigación, por el período establecido para el desarrollo del trabajo de campo. Finalmente se concluye que las principales necesidades educacionales y formativas son: lenguaje corporal-vocal, género y alfabetización crítica. Por otra parte, tras la carencia de articulación entre las instituciones públicas encargadas de velar por el mejoramiento de dicho centro educativo, se recomienda la creación de un Departamento de Educación o Mediación Artística encargado de velar por la articulación de los distintos entes institucionales públicos para que se dé apertura al mejoramiento de los estatutos, normativas y plan de estudio de la APNAE, y de este modo se contemple la estrategia de pedagogía teatral desde un diseño universal.
\end{abstract}

Palabras clave: diversidad funcional, educación inclusiva, pedagogía teatral, necesidades educacionales

\begin{abstract}
The present text sets out the objective of identifying the current training needs, as well as the pedagogical-administrative challenges and scopes, that implies the updating of the curriculum so that the theatrical pedagogy is integrated as a regular subject in the Association for Children and Exceptional Adults (APNAE), therefore, the current state of Inclusive Education and Artistic Education in Costa Rica is contextualized. Then, its expose the conceptual axes of the research, which give way to the construction of the analysis categories that are explained in the qualitative methodology, in which a participatory diagnosis was executed with the three study groups. The limitations of this research are that the institutional body (CONAPDIS, Department of Educational Supports for students with disabilities of the Ministry of Education, people or entities of Ministry of Culture and Youth) was not integrated as part of the research subjects due to a time limitation in the inquiry progress. Finally, it is concluded that the main pedagogical needs are: body-vocal language, gender and critical literacy. On the other hand, after the lack of articulation found between the public institutions in charge of ensuring the improvement of the educational center, it is recommended the creation of an Artistic Education Department in charge of ensuring the articulation of the different public institutional entities for the improvement of the statutes, regulations and curriculum of the APNAE, and in this way the theater pedagogy strategy is contemplated from a universal design.
\end{abstract}

keywords: functional diversity, inclusive education, theater pedagogy, educational needs

\footnotetext{
1 Profesional Independiente, Costa Rica. Licenciada en Administración de la Educación no formal, Universidad de Costa Rica. Dirección electrónica: melissa.rodriguezrios@ucr.ac.cr ORCID: https://orcid.org/0000-0003-1016-4271
}

Artículo recibido: 24 de octubre, 2019

Aprobado: 27 de abril, 2020 


\section{Introducción}

Pensar la Pedagogía Teatral bajo el modelo de la Educación Inclusiva en el sistema educativo formal costarricense, representa cada vez más una necesidad así como una oportunidad en las aulas; una necesidad en tanto para las personas docentes como para el estudiantado y su entorno familiar, caracterizándose el espacio de encuentro (aulas), por dinámicas que no contemplan las múltiples maneras de aprender, además de factores conductuales de la cultura en sí, que se ha ocupado de dividir, "etiquetar" y sostener una estructura de enseñanza-aprendizaje que ha llegado a caducar. Por ello, se considera una oportunidad que la Pedagogía Teatral sea un medio que contemple entrenar y reconocer las distintas inteligencias que la persona posee, mediante un diseño que sea accesible para todo el estudiantado.

Previo a profundizar otros aspectos contextuales de la investigación, conviene aclarar las partes que componen este artículo, el cual se conforma por cuatro ejes, siendo esta la primera, en la cual se contextualiza sobre el tema, y, por ende, el estado de la Educación Inclusiva y de la enseñanza de las artes dramáticas, es decir, de la pedagogía teatral en el sistema educativo formal del país. En la segunda parte se desarrolla el marco conceptual de la investigación la cual se sustenta de los siguientes ejes conceptuales: diversidad funcional (personas con discapacidad), las necesidades formativas (necesidades educacionales), la educación por el arte (como área específica de la pedagogía teatral) y la educación inclusiva. La tercera parte responde a la metodología de la investigación realizada, en la cual da continuidad al marco conceptual por medio de la operacionalización de los objetivos (categorías de análisis), los cuales de forma transversal en la investigación permiten dilucidar retos y alcances a partir de indicadores de cada categoría, respectivamente. Por último, en la cuarta parte se expone un análisis crítico de los alcances y retos entorno a la Educación Inclusiva y la Pedagogía teatral en el Centro de Atención Integral Para personas con Discapacidad (CAIPAD en adelante) de la Asociación Pro Niño y Adulto Excepcional (APNAE en adelante), así como posibilidades de considerarse esta última como modelo en otros CAIPAD del país.

En continuidad con la necesidad y contextualización de esta investigación, emergen las distintas barreras que siguen obstaculizando la viabilidad artística en escenarios educativos con mayor inequidad y exclusión social, como es el caso de las personas adultas con 
diversidad funcional que asisten a la $\mathrm{APNAE}^{2}$ el cual está vinculado a los $\mathrm{CAIPAD}^{3}$, los cuales forman parte del Ministerio de Educación Pública (MEP en adelante). El aporte de dicha institución pública es mediante recurso humano para suplir las necesidades pedagógicas, por lo que se asigna a este CAIPAD cuatro docentes (una del área de Educación Especial quien asume la coordinación del equipo base, el cual está integrado por personal del campo de rehabilitación, terapia ocupacional y del área social); según Alpízar et al., "De ahí que un requisito fundamental para operar un CAIPAD es regirse por el plan de estudios aprobado por el Consejo Superior de Educación" (2013,p.10).

Por ende, conviene aclarar el estado de la educación inclusiva como parte de las políticas internacionales en derechos humanos, con el fin de esclarecer sus transiciones y evoluciones hasta el presente siglo XXI y su práctica en contextos educativos como el CAIPAD APNAE. El paradigma de la educación inclusiva se ha ido definiendo a lo largo de la historia, en Costa Rica, a partir de la Declaración Mundial de Educación para Todos, realizado en Jomtiem-Tailandia 1990 convocada por la UNESCO, en donde se gestó el proyecto UNESCO/ACDI en 1992. Así que Costa Rica realizó el Plan Nacional de Acción para mejorar las metas propuestas en Jomtiem antes del 2000. Sin embargo, según la UNESCO, en Dakar 2000, "los logros fueron escasos, pocos propósitos se acercaron a la meta y en muchos casos los países más pobres del mundo se encontraban en peores condiciones que las diagnosticadas en Jomtien (1990)" (p.60).

Varela (s.f) cita el Programa del Estado de la Nación 2013 para rectificar que, en el plano educativo, la desigualdad de oportunidades persiste en Costa Rica desde diversas áreas sociales, étnicas, culturales y territoriales. Es por esto que el fenómeno de la inclusión excluyente es una problemática compleja que remite a varios factores. Además, Varela (2015) menciona que el programa curricular del proyecto educativo nacional, cuya condición es estandarizada, se vuelve social y culturalmente inequitativa y excluyente, porque no es accesible de la misma manera para todos los sectores, grupos y diferentes poblaciones estudiantiles. Ejemplo de ello, es el marco de la Educación Especial en el contexto costarricense, cuya modalidad pedagógica es mediante la adecuación curricular, o bien, las

\footnotetext{
2 Según el APNAE (2011) en el reglamento interno se define la APNAE como: “... una Asociación Herediana comprometida con la formación y el desarrollo integral de las personas con alguna condición de discapacidad” (p.4).

${ }^{3}$ Los centros CAIPAD es un servicio para las personas con discapacidad mayores de 21 años, que requieren de apoyos prolongados o permanentes, y se ofrecen procesos de alfabetización, participación social, ocupacional y laboral, que les permita potenciar su desarrollo integral, su autonomía personal y mejores condiciones de vida. Por medio de un equipo de trabajo, comprometido y capacitado en derechos humanos (Alpízar et al., 2013, p.51).
} 
llamadas adecuaciones no significativas, que generan un sesgo importante desde la concepción de la educación para todos. ¿Por qué las adecuaciones curriculares generan un sesgo? Como bien lo remite su nombre, la educación para todos pretende que exista un diseño educativo, que resulte accesible para los grupos de personas tomando en cuenta variables como: contexto, ritmos de aprendizaje, conocimientos aprendidos según su nivel (escolar) o bien, etario. Lo anterior no significa que dicho diseño universal no pueda ser flexible ante las necesidades de aprendizaje de un grupo de estudiantes que así lo requieran, contrario, a como ocurre en las adecuaciones curriculares, en donde se aplica la distinción y programación de un diseño curricular para aquella persona que necesita de apoyos. Mientras que, en el diseño universal se programa tomando en cuenta los apoyos que la persona con necesidades de aprendizaje o con alguna condición de discapacidad va a requerir, para que pueda plenamente transitar un diseño curricular con autonomía y seguridad, al igual que las demás personas no poseen alguna condición. Por lo tanto, en el diseño universal, según Labarga (comunicación personal, 11-13 de setiembre, 2019) "no existen habilidades medias, hay posibilidades diversas".

Existe un contexto del por qué las estrategias pedagógicas desde las artes escénicas no se han implementado de forma regular en centros educativos destinados para personas adultas con diversidad funcional, a pesar que se estipula en el título II, capítulo VII de la Ley $\mathrm{N}^{\circ} 7600$, el derecho al acceso a la cultura, el deporte y las actividades recreativas para la población con discapacidad en igualdad de oportunidades (2015, p.10). Aunque a su vez, dicho título se ve limitado a una interpretación unidireccional, es decir, siendo las personas con diversidad funcional receptores o espectadores de cultura, en lugar de ser partícipes, es decir, personas creadoras de las distintas disciplinas artísticas y recreativas que integran el ámbito cultural. A pesar que, la Convención sobre los derechos de las personas con discapacidad, compendio Ley 8661 (2009), menciona en el artículo 30, punto 2, que "Los Estados Partes adoptarán las medidas pertinentes para que las personas con discapacidad puedan desarrollar y utilizar su potencial creativo, artístico e intelectual, no sólo en su propio beneficio sino también para el enriquecimiento de la sociedad" (p.23). Aun así, la realidad contextual se ve reflejada en el informe del Instituto Nacional de Estadística y Censos (2018), en la cual se identifica la participación de las personas mayores de 18 años con alguna condición de discapacidad en relación a actividades artísticas y recreativas. Dicho porcentaje respecto a la población nacional es de un 18,2\%, correspondiendo a un $30 \%$ de personas con discapacidad que practican alguna actividad artística, mientras que, la asistencia a 
eventos culturales y deportivos por parte de personas con alguna discapacidad es de un $68,9 \%$.

Los anteriores datos, permiten dar una noción de cuántas personas con diversidad funcional están accediendo a las actividades artísticas específicamente, dado que es el interés de esta investigación la pedagogía teatral, como estrategia artística en la educación. Sin embargo, una dimensión estructural que dificulta la operacionalización de la misma en diversos centros educativos del país, se debe a que “...estas políticas a favor de la educación artística como programas de educación no formal no se han concretado hasta la actualidad tras la carencia de políticas del gobierno para la Educación Artística no formal" (Collado, s.f, p.1).

La problemática radica en que hay un retraso significativo sobre la transversalización de modelo de educación inclusiva y de la inserción de la pedagogía teatral al currículo educativo, tal y como Benavídez (s.f) menciona: "En este siglo, en que los avances en la educación artística son notables, es lamentable que todavía estos no traspasen los muros de las instituciones educativas regulares y especiales en pro del desarrollo integral del alumno (a)" (p. 44).

Resulta fundamental, esclarecer el papel que el CAIPAD APNAE tiene en este tema, dado que el mismo se encuentra en un momento histórico en el cual su modelo de integración educativa no se ha replanteado hacia el modelo de Educación Inclusiva, quizá por los retos administrativos y pedagógicos que estos conllevan. Ejemplo de lo anterior se puede identificar con la meta educativa que se propone el plan de estudios del CAIPAD APNAE, según Alpízar et al., (2013) se plantea “...que la mayor cantidad de personas que han ingresado a un CAIPAD, puedan en un tiempo determinado estar ubicadas en un empleo remunerado, desarrollando un proyecto productivo familiar/comunal o bien una actividad laboral participativa" (p.9). Paralelamente, los programas educativos de habilidades para la vida, se implementan para dar apoyo a las necesidades básicas de estas personas en su interacción cotidiana con su entorno social particular. Además, es importante resaltar que cada CAIPAD ${ }^{4}$ desarrolla su plan de estudios anualmente, con plena independencia según las prioridades que el equipo de docentes contemple, siendo el área de educación

${ }^{4}$ En el país existen 28 CAIPAD en distintas zonas. 
física por ejemplo, una inversión gestionada por parte de las Juntas Directivas ${ }^{5}$, y no del Estado.

Con base a los anteriores antecedentes, y la no vinculación de la educación corporal desde las artes en el diseño curricular del CAIPAD APNAE en específico, se formula la siguiente pregunta de investigación: ¿Cuáles son las principales necesidades educacionales o formativas de la población adulta con discapacidad del APNAE CAIPAD, y cuáles estrategias pedagógicas y apoyos son pertinentes, para que dicho medio pedagógico pueda vincularse como modelo en otros CAIPAD del país?

Aunque, en la gestión de procesos socio educativos no formales, es complejo hablar de pasos, dado que los mismos dependen de un conjunto de elementos y factores que posibilitan o no su programación educativa. He ahí los retos y alcances de la programación un plan de estudios que contemple la educación corporal desde las artes con enfoque inclusivo.

\section{Base teórica de la investigación}

Al tratarse de una investigación situada, las personas con discapacidad de la APNAE se convierten en objeto de estudio de forma transversal, debido a las implicaciones que la incidencia de la construcción social que esta definición ha tenido en la identidad personal y sociocultural de personas con diversas condiciones. Tal y como se refiere Chaves (2001) el desarrollo humano está íntimamente ligado con su interacción en el contexto sociohistóricocultural, siendo la educación el medio de desarrollo potencial del sujeto (p.59). Al tomar en cuenta la definición de Verdugo (1997) citado por Shalock (1999) sobre la discapacidad, él remite que "no es algo fijo ni dicotomizado; preferentemente, es algo fluido, continuo y cambiante, dependiente de las limitaciones funcionales de la persona y de los apoyos disponibles en su entorno" (párr.12). Por lo que en la actualidad el entendimiento de la discapacidad se debe realizar desde una perspectiva contextualizada con un modelo ecológico de discapacidad basado en la interacción del individuo con su contexto.

Desde los estudios críticos de la Teoría Crip, surge el aporte de la diversidad funcional, término que pretende un abordaje y cuestionamiento conceptual de la discapacidad. Fernández (2016) menciona que dicha definición es “...una vía de auto reconocimiento que

\footnotetext{
${ }^{5}$ La nómina de personas colaboradoras y participantes de la asociación a través de la Junta Directiva, según el Reglamento de Conducta (2011) son: siete padres, madres o personas encargadas de los adultos en condición de discapacidad, los cuales toman decisiones importantes para el manejo adecuado de esta institución.
} 
se contrapone al carácter estigmatizante que conlleva el término "discapacidad" (p.2). Por otro lado, la educación holística realiza su aporte conceptual sobre la funcionalidad, la cual implica según Maya (2013) “...reconocer el potencial innato de cualquier estudiante para el pensamiento inteligente, creativo y sistémico", además, "reconoce que la inteligencia es multidimensional y puede ser expresada y valorada en una variedad de formas diferentes de las meramente matemáticas y verbales...” (p.245).

Por otra parte, la diversidad “...nos remite al hecho de que todos los alumnos tienen unas necesidades educativas individuales propias y específicas para poder acceder a las experiencias de aprendizaje necesarias para su socialización, que están establecidas en el currículo escolar. Estas necesidades educativas individuales tienen su origen en las diferencias culturales, sociales, de género y personales" (Centro Nacional de Recursos para la Inclusión Educativa, s.f, p.3).

A partir de los anteriores referentes conceptuales, se reconoce la complejidad humana y las potencialidades en sus modos de aprendizaje, más que posicionarse en la condición de discapacidad predominante. Por ende, el abordaje es al inverso de como usualmente se han pensado los procesos educativos para esta población en específico. Siendo así que, la Teoría Crip se propone descolonizar el capacitismo corporal "(la idea de que todos los cuerpos deben describir el mismo repertorio de funciones y capacidades)" (p.82), ligado también a modelos económicos y políticos hegemónicos que inciden en la construcción de la identidad a través del lenguaje, el cual trae valores culturalmente aceptados, más aún, en marcos legales, validados por entes de poder (instituciones), mientras que la diversidad funcional, según Brugarolas (2015) no está validado legalmente ni extendido internacionalmente. Esto tiene una explicación histórico cultural, en la cual la terminología para nombrar a las personas con discapacidad, se ha ido transformando a medida que la cultura de cada sociedad produce formas de comportamiento, es decir, creencias y prácticas, que se constituyen por marcos o estructuras que se componen de un discurso, o bien, de un relato figurado por la historia, tal y como lo menciona (Lagarde,M, comunicación directa, 1113 de setiembre, 2019).

En nuestro presente, la terminología de la diversidad funcional en la cultura costarricense no es aún reconocida, estamos adaptándonos a palabras que se han validado desde varios años atrás por parte de un modelo y directriz macro, sin embargo, en lo micro (algunos movimientos sociales, organizaciones, academias, entre otros), hay una necesidad de ampliar, transformar las palabras y, por ende, el sistema de creencias. Desde este estado 
de cuerpos en resistencia, se defiende con el término de diversidad funcional la particularidad conceptual de que "nuestros límites no son nuestras fronteras corporales..." (Fregtman, 1985, citado por Maya, 2013, p.251).

Ligado a la diversidad funcional, el modelo de la Educación Inclusiva aporta algunos elementos fundamentales para la construcción de procesos de aprendizaje en correspondencia a la anterior definición. Algunas de las dimensiones que integran este modelo son: los apoyos y barreras, las comunidades de aprendizaje, los ritmos de aprendizaje y la gestión de procesos socio educativos.

Si bien, la visión de la UNESCO en la Declaración Mundial de Educación para Todos formulada en Jomtien, la cual se confirma en el Marco de Acción de Dakar (2000), en ella se expresa que: "el compromiso colectivo de la comunidad internacional de perseguir una estrategia amplia, con objeto de garantizar que en el lapso de una generación se atiendan las necesidades básicas de aprendizaje de todos los niños, jóvenes y adultos y que esa situación se mantenga después" (p.12). Del mismo modo lo afirma Blanco (2002), quien menciona que para que se posibilite la Educación Inclusiva se debe eliminar las barreras que existen para el aprendizaje y la participación de muchos niños, jóvenes y adultos, con el fin de que las diferencias culturales, socioeconómicas, individuales y de género, no se conviertan en desigualdades educativas y, por esa vía, en desigualdades sociales (p.22). Para ello, las comunidades de aprendizaje son estrategias de organización que la Educación Inclusiva ha desarrollado para la equidad entre distintos actores sociales.

Para Santos (2000) las comunidades de aprendizaje tienen el objetivo de "...conjuntar todo aquello que puede contribuir a romper las dinámicas negativas de una situación, favoreciendo la relación entre un centro educativo y su entorno, pero también la relación entre los miembros de la comunidad escolar" (p.140). Por lo tanto, las comunidades de aprendizaje “...son el resultado de la transformación social y cultural de un centro educativo y de su entorno mediante una educación integrada, participativa y permanente mediante ese tipo de aprendizaje" (Santos, 2000, p.141). La función de las comunidades de aprendizaje es promover el acceso, al contemplar la mediación pedagógica como estrategia para el manejo de los grupos de aprendizaje y sus actividades, permitiendo que las necesidades formativas se personalicen o se aborden de forma colaborativa.

La anterior estrategia se relaciona con la educación por el arte, la cual es un área de la pedagogía teatral, definida por Astrosky (setiembre del 2015, comunicación directa modalidad virtual) como el uso de actividades artísticas para desarrollar las cualidades 
personales, sin tratar de formar profesionales de un área, por lo que se busca enriquecer a la persona tanto en su conocimiento como en su desarrollo, teniendo como recurso a las actividades artísticas, no sólo en cuanto a la especificidad de los contenidos, sino también a socialización de lo grupal y el trabajo sobre cierto tipo de valores (cooperación, compañerismo, solidaridad, etc).

La importancia de la pedagogía teatral como eje de investigación radica en que en sus bases epistemológicas se haya el enfoque histórico cultural de Vygotski, siendo el principio social de este enfoque, según Chaves (2001) lo que hace que el sujeto no se desarrolle solo por sí mismo, sino que todo su entorno es un sistema social que está compuesto por la colectividad y la comunicación que surge de la misma dinámica (p.60). Dicho procedimiento anterior difiere con el proceso natural o biológico, debido a que, al surgir el lenguaje como resultado de la interacción social y afectiva, se provoca interiorización en la persona, por ende, se genera una manifestación entre la palabra, el gesto, la acción, la sensación, el movimiento, entre otros. Estas manifestaciones del lenguaje, permiten develar dos niveles: el nivel actual de desarrollo y la zona de desarrollo próximo (ZDP), lo que permitirá identificar formas y ritmos de aprendizaje, capacidades en torno a la autonomía corporal, entendido por Castillo (2012) como un proceso de autodescubrimiento y a su vez del grupo, en el que se favorece la comunicación creativa, el control mutuo, el cuestionamiento y la auto organización corpórea y creativa.

Las anteriores dimensiones conceptuales que atañen a esta investigación se intersecan por medio del estudio de las necesidades educativas, entendida desde la construcción de procesos de educación no formal, como necesidades formativas, según Baraibar et al., (2003) son "...la discrepancia entre la situación actual de una persona o grupo y la situación considerada como meta o referencia" (p.111). Para Benavides (s.f) dicha meta educativa debe de estar fundamentada en las necesidades educativas y en los deseos del estudiantado, lo cual no es una realidad en la mayoría de las instituciones educativas (p.45). Sobre la anterior afirmación, se reafirma una postura personal hacia la investigación cualitativa en que la misma debe de contemplar las distintas realidades y lecturas que una situación o circunstancia social presente, para lo que resulta una posibilidad, que el enfoque participativo como metodología cualitativa da a conocer las subjetividades de las personas implicadas. Al respecto se profundiza en este apartado a continuación.

\section{Metodología de la investigación}




\subsection{Enfoque}

El enfoque de investigación que se realizó fue cualitativo, siendo su alcance descriptivo y exploratorio. El diseño de la investigación se caracterizó por el empleo del diagnóstico participativo de forma transversal para poder valorar los fenómenos sociales, culturales, contextuales y corporales de las personas de estudio, por lo tanto, se entiende por diagnóstico participativo según Aguilar, Briceño y Valenciano (1999) un “...proceso sistemático que sirve para reconocer una determinada situación y el porqué de su existencia, en donde la construcción del conocimiento se hace con la intervención y opiniones de las personas que tienen que ver con esa situación" (p.11). Por ende, el alcance del diagnóstico permitió determinar las necesidades educacionales de las personas que asisten a la APNAE, a partir de los hechos observados y su interpretación.

\subsection{Unidades de análisis}

La población participante se definió en tres grupos de estudio: el primer grupo conformado por cuatro docentes del APNAE, el segundo grupo por cuatro personas de núcleos familiares de estudiantes de la APNAE (dos de ellos pertenecientes a la Junta Directiva) y, por último, con doce personas con distintas condiciones de discapacidad (personas con Síndrome de Down, discapacidad intelectual leve y medio, y discapacidades motoras), los cuales se dividieron en dos grupos de trabajo. Dicha selección de la población participante responde a criterios de inclusión y exclusión de la investigación.

Algunas de las consideraciones éticas que se realizaron para el empleo de esta metodología fue el consentimiento por parte de las personas responsables de las personas adultas con diversidad funcional para que pudiesen participar en los talleres con fines investigativos. Además, se solicitó un consentimiento exclusivo para el uso de imágenes (fotografías y video con fines de sistematización de la información). Del mismo modo se solicitó con consentimiento a las personas docentes y núcleos familiares de personas con diversidad funcional que participaron. A todas las personas se les respetó su confidencialidad para la interpretación de la información.

Los criterios de inclusión de la metodología de investigación consistieron en: involucrar a aquellas personas que quisieran colaborar del proceso de indagación de forma comprometida, personas que tuvieran al menos dos años de permanecer en la institución, personas con cualquier condición de discapacidad y con distintos rangos de edad. Los criterios de exclusión fueron que aquellas personas que participaran de los talleres no 
presentaran alguna condición significativa (lesión, recuperación operatoria, estado anímico que le impidiera estar durante la sesión práctica, entre otros). Por otra parte, por recomendación de la directora de la institución, se sugirió no trabajar con personas no oyentes debido a que no manejan el lenguaje LESCO.

La indagación se realizó durante el 2018, en un periodo de recolección y análisis de la información de ocho meses aproximadamente.

\subsection{Técnicas de recolección}

Las principales técnicas de recolección de la información fueron: la observación participante, talleres y entrevistas semiestructuradas.

Dicho procedimiento se dividió en dos fases: la primera fue de indagación del contexto institucional y del marco conceptual, la fase de desarrollo se ejecutó con tres grupos de personas: el primer grupo estuvo conformado por doce personas adultas con diversas condiciones de discapacidad cognitiva, de este equipo se conformó dos subgrupos de seis personas cada uno. Con cada subgrupo se realizaron cinco sesiones de taller con técnicas de gestalt, movimiento somático, juegos de integración, construcción de líneas de vida y mapas o cartografías corporales. Entre los principales recursos utilizados se encuentran: los pictogramas, mapas corporales, los estímulos sonoros y el cuerpo, principal medio de investigación.

La segunda población de investigación fue definida por dos personas de los núcleos familiares del estudiantado y dos personas que además de ser parientes tuvieran un rol activo en la Junta Directiva del APNAE. La elección de este grupo de investigación responde a la necesidad de identificar y valorar el grado de conciencia de las necesidades educativas, en primer lugar, de núcleos familiares hacia su pariente (estudiante del centro), y hacia la generalidad del estudiantado que asiste al APNAE, y, en segundo lugar, de personas que poseen un cargo administrativo, en el cual se ven permeados de información y toma de decisiones para el mejoramiento del centro educativo.

El último grupo de población se delimitó a las cuatro docentes asignadas por el MEP, a quienes se les realizaron entrevistas con un instrumento semiestructurado. Además, durante cada encuentro se realizó observación participante mediante una guía de observación la cual contenía las siguientes dimensiones: mediación pedagógica, las necesidades de aprendizaje de las personas con diversidad funcional, motivaciones o expectativas de la población, la 
comunicación verbal y no verbal del estudiantado, y, por último, las barreras y apoyos del entorno y de las personas según sus condiciones.

\subsection{Procesamiento de análisis}

El análisis de la información se realizó mediante las siguientes categorías de análisis y su procesamiento fue con un análisis descriptivo. Las categorías de análisis para el procesamiento de dicha información fueron: diversidad funcional, educación no formal, educación por el arte y educación inclusiva; siendo sus variables:

- Diversidad funcional: comprensión de la discapacidad, paradigmas, necesidades de aprendizaje, las motivaciones personales y la mediación pedagógica que se requiere según la necesidad.

- Necesidades formativas: en esta categoría no se definieron indicadores debido a que los hallazgos iban a determinar cuáles necesidades de formación se identificaron.

- Educación por el arte: autoimagen y autonomía corporal.

- Educación Inclusiva: paradigma en Derechos Humanos, Modelo de Integración Educativa, métodos cooperativos, comunidades de aprendizaje y barreras y apoyos.

A partir de los hallazgos obtenidos de las anteriores dimensiones y sus respectivas variables, se encontraron variables emergentes como: ritmos de aprendizaje y la gestión de procesos socio educativos desde la educación no formal. Siendo los principales alcances de la metodología empleada, el reconocimiento de necesidades formativas de forma integral, en donde se integró el cuerpo y sus discursos, el cuerpo y sus posibilidades, las contradicciones ideológicas, las dificultades estructurales y socio culturales en la educación. Al ser una investigación participativa, la información recolectada e interpretada se validó con las personas que formaron parte de la indagación. Por ende, un alcance de la metodología es su cualidad de ser reinterpretada según las distintas realidades de las personas sujetas de investigación.

\section{Resultados}

\subsection{Limitantes y alcances en el devenir de la Educación Inclusiva del CAIPAD APNAE}

Para comprender el modelo de gestión educativo del CAIPAD APNAE, desde los fundamentos de la educación inclusiva, es importante reconocer que el origen de esta institución estuvo bajo la influencia del modelo de integración educativa, el cual correspondió 
a un paradigma tradicional y rehabilitador. Además, de que su gestión administrativa estuvo y se encuentra aún liderada por familias de personas con diversidad funcional, quienes buscaban una alternativa de educación para adultos.

Las consecuencias de las aulas integradas que se implementaron también en dicho momento histórico de la Integración Educativa, se ha logrado dimensionar en la actualidad a través del CAIPAD APNAE, quienes son resultado de segregación educativa, social y laboral.

Problematizar la educación inclusiva en el contexto costarricense representa un reto por el cambio epistemológico y de gestión administrativa que esta conlleva. En cuanto a lo epistemológico, el modelo social de la discapacidad es quizá el enfoque que tiene más similitudes con la diversidad funcional, aunque, a su vez, desde el movimiento de Teoría Crip (donde surge el concepto de diversidad funcional), se critica el asistencialismo que ha replicado el modelo social de otros modelos, por ende, desde la Teoría Crip reivindica que la transformación debe ser cultural para que la estigmatización deje de reproducirse y con ello, los hábitos institucionales y sociales para las personas con diversidad funcional. Un hallazgo con relación a lo anterior, es la dependencia hacia beneficios económicos (becas) que reciben mensualmente las personas con diversidad funcional, por lo que la mayoría de las familias prefieren que permanezcan de este modo en lugar de que logren una autonomía económica con un puesto laboral, de los cuales eventualmente podrían ser despedidos o bien, desertar en caso que la persona con diversidad funcional no logre adaptarse en el entorno profesional.

Otro hallazgo sobre las barreras que se normalizan por parte de los núcleos familiares y docentes, son las denominaciones que se utiliza para caracterizarlos y categorizarlos a partir de sus enfermedades o patologías. Además, de formas para nombrarles a través de palabras que infantilizan a la población adulta con diversidad funcional, o bien, frecuentando calificativos entorno a sus almas o personalidades "tan buenas". También, se encuentra que sus edades llegan a ser una barrera para aprender algo nuevo.

Por lo tanto, la discapacidad desde el paradigma tradicional, se ha instalado en los entornos familiares y en las mismas personas con necesidades de aprendizaje diferentes, alimentando un discurso que se justifica en la enfermedad o en las patologías diagnosticadas como limitantes de los aprendizajes.

Siendo, los núcleos familiares uno de los factores que limita el desempeño e independencia de las personas con diversidad funcional, aunque no debe obviarse que este núcleo es reforzado por el entorno social, mismo que a su vez ha generado que parte del 
estudiantado se identifique como victimario. Así mismo, el prejuicio y hostilidad se ve reflejada entre el estudiantado de la APNAE a causa de las distintas funcionalidades que cada quien posee. Lo anterior se ve expuesto entre las personas que pasaron por procesos previos de educación y quienes no tuvieron la oportunidad de atravesar procesos formativos, aunque, no todas las personas lograron los resultados esperados en las aulas integradas (lecto escritura, por ejemplo).

Para las docentes lo anterior implica una limitante educativa que ha tenido esta población desde la primera infancia (con destrezas de estimulación temprana hasta de alfabetización), por distintos factores como: poco o nulo acceso a la educación, carencia en prácticas pedagógicas acordes a las necesidades educativas de la persona, condiciones cognitivas de mayor complejidad en la persona, poco compromiso familiar hacia los apoyos requeridos por la persona, y segregación del entorno social.

Por otro lado, las características que el centro posee (al ser exclusivo para personas con diversidad funcional) generará un reto para cualquier estrategia pedagógica que se desee plantear desde un enfoque inclusivo, sin embargo, resulta importante validar otros indicadores de inclusión (además del nivel de participación de personas con y sin diversidad funcional), como por ejemplo: estrategias alternativas para vincular a las personas con diversidad funcional con la comunidad, propuestas educativas innovadoras para sus aprendizajes, contenidos de aprendizaje pertinentes y que impliquen una deconstrucción de la discapacidad (desde los diversos paradigmas), empoderamiento para la incidencia política de sus derechos y por ende, de su autonomía económica. Sin embargo, volviendo a los principios de la Teoría Crip, la transformación debe ser cultural, por lo que debe de haber un cambio estructural. Al respecto, se reconoce que existen diversas limitantes relacionadas a la administración educativa que imposibilitan avances en cuanto a inclusión social. Aun así, algunos alcances que podrían plantearse para la transformación educativa del CAIPAD APNAE son:

- Integrar en la administración (Junta Directiva) a personas profesionales en educación.

- Generar mayor co-responsabilidad con el Estado, para que incida con la mejora de la infraestructura según las necesidades (apoyos pertinentes).

- Actualización del plan de estudios, así como de los estatutos de la CAIPAD APNAE (objetivos, visión y misión) de forma integral y participativa.

- Monitoreo del adecuado funcionamiento del reglamento institucional por parte de la Junta Directiva y de otras instituciones estatales. 
- Contratación de más personal docente que esté capacitado, y a su vez aporte con innovación pedagógica en las diversas áreas que se requieren.

- Conformar un comité integrado por núcleos familiares, docentes y otras instituciones para velar por proyectos autogestionarios que financien necesidades del centro educativo, así mismo, dichos espacios podrían ser espacios de capacitación y de organización o cohesión interna.

- Vincular al estudiantado mediante proyectos formativos comunitarios, en donde puedan tejer relaciones sociales de forma inclusiva.

- Activar el Comité de Capacitación de la institución CAIPAD APNAE con todos aquellos procesos educativos formales y no formales, en el cual se logre planificar, evaluar y sistematizar los aprendizajes constantemente.

- Tomar en cuenta el diseño universal para el planeamiento anual y metodológico, el cual es accesible para cualquier persona y no solo para quienes tienen una condición específica.

\subsection{Limitaciones y alcances de la innovación educativa a través de la educación por el arte (dimensión de la pedagogía teatral)}

La metodología de investigación puso su mirada en la corporalidad, en tanto percepción propia y del otro. Para Gallagher (2005, citado por Álvarez, 2014) la autoimagen es "...como un (a veces consciente) sistema de percepciones, actitudes, creencias y (pre) disposiciones que pertenecen al propio cuerpo de cada uno/a" (p.17). Mientras que para Aguado (2004) la imagen corporal se define como “...una estructura tridimencional que permite la conciencia del sí-mismo integrando los aspectos físicos, estructurales y fisiológicos en relación con el movimiento (por lo que incluye el tiempo). Es una estructura que integra las sensaciones, las emociones y la percepción, por ello es la base de la experiencia en la que se integra el significado cultural". (p.49, citado por Hidalgo, 2015, p.90).

Siendo la noción de cuerpo algo cambiante para cada persona, en el caso de Álvarez (2014) "las cualidades propias de la imagen pueden ser expresadas de manera visual e interpretadas como algo que te pertenece, que posees: por ejemplo, "me veo grande..." (p.20). Por lo que Pylvänäinen (2003, citado por Álvarez, 2014), menciona que este aspecto corporal es al que más se refiere nuestra sociedad, lo que reduce el concepto de la imagen a algo superficial, por lo que resulta fundamental para una educación corporal desde las artes un proceso transformativo, para llegar al yo corporal, entendido por Álvarez (2014) como un 
cuerpo vivido, la esencia, en vez de lo que posee. He ahí la importancia de la educación por el arte (según categoría de la pedagogía teatral en la que se concibe el arte como medio y no como fin en sí mismo), como estrategia pedagógica que produce cambios en la imagen corporal.

Con base a la anterior premisa de investigación, los resultados obtenidos a partir de la metodología se sistematizaron en función de la categoría de educación por el arte, que a su vez contempló el lenguaje corporal y su comunicación como un elemento fundamental para poder determinar ritmos de aprendizaje, los apoyos y barreras que cada persona participante requiere, la mediación pedagógica que se considera necesaria para facilitar desde este campo de saber, y finalmente, las necesidades formativas (de la propia disciplina artística como también de áreas sociales).

Por ende, resulta pertinente ahondar en el concepto de dicha estrategia. Para Boal (2001) citado por Arboleda et al. (2011) la función del teatro es la de traer felicidad, además, “...debe ayudarnos a conocer mejor nuestro tiempo y a nosotros mismos. Nuestro deseo es conocer mejor el mundo en el que vivimos para poder transformarlo de la mejor manera. El teatro es una forma de conocimiento y debe ser también un medio de transformar la sociedad. Puede ayudarnos a construir el futuro, en vez de esperar pasivamente a que llegue..." (p.27). Entender la relación entre teatro y educación para personas con diversidad funcional, supone otra especificidad conceptual. Según García (1996), en dicha disciplina, la pedagogía teatral es un marco de acción y de orientación psicodinámica y comunitaria que facilita la toma de conciencia de las propias dificultades y limitaciones, sin embargo, permite al mismo tiempo generar vínculos entre la persona y su medio ambiente, complementando habilidades adquiridas para potenciar su capacidad laboral y su posible inserción en la sociedad (pp.10-11).

Por lo tanto, ¿qué aporta la estrategia de pedagogía teatral y con qué limitaciones se encuentra?

Las principales limitantes que intervienen para operacionalizar esta estrategia, son los mecanismos de entes rectores encargados de diseñar e implantar políticas públicas. Así como lo afirma, Meléndez (2019) "En la totalidad de los países de América Latina existen instituciones con instrumentos legislativos y de políticas públicas específicas, abocadas a hacer cumplir los derechos de las personas con discapacidad y a diseñar e implementar políticas públicas que materialicen la inclusión y participación social plena de este grupo poblacional" (p.10)." No obstante, son pocos los países que cuentan con un sistema de 
información y de indicadores que permita dar seguimiento a estas políticas públicas (Ullmann, 2017, p.293, citado por Meléndez, 2019, p.13).

En este aspecto, he la importancia de que el Departamento de Educación Especial del MEP evalúe las actuales estrategias pedagógicas y las necesidades formativas demandadas con el fin de validar otras áreas de conocimiento distintos a los enfoques rehabilitadores, sensibilizando paralelamente a los actores sociales que forman parte de la institución, para que se considere una prioridad la innovación educativa, y por ende, se asigne otros mecanismos para captar presupuesto para beneficiar las distintas necesidades formativas.

Otros alcances que se podrían dimensionar en un futuro para el CAIPAD APNAE a partir del área de conocimiento de las artes escénicas son:

- Transdisciplinariedad en las formas de enseñanza-aprendizaje entre el equipo de docentes.

- Visibilizar en el sistema educativo formal que las artes escénicas a través de la auto imagen corporal y la creatividad, disminuye bloqueos y somatizaciones corporales (las cuales responden a mecanismos transpersonales y socioculturales de la persona) y no meramente a una patología, tal y como lo plantean otros paradigmas. Dichos bloqueos o corazas son definidos por la teoría de la coraza de Reich, para quien el principal mecanismo de defensa ante el temor es la represión corporal. Según De León (s.f) se trata de una inevitable disminución de la movilidad psíquica, formándose por medio de bloqueos de segmentos, que se manifiestan como defensas del ego llegando a ser crónicamente activas y automáticas, y, por ende, transformándose en rasgos del carácter, como mecanismos de protección que se han hecho crónicos en la conducta y el cuerpo (grupos musculares crónicamente tensos que bloquean la energía" (p. 3). Además, "Las defensas del carácter son particularmente efectivas e igualmente difíciles de erradicar porque están bien racionalizadas por el individuo y son experimentadas como parte de su auto concepto y tienen su representación en el cuerpo" (De León, s.f, p.3). Por lo cual, la rigidez es la base de la reacción característica y su emergencia se debe a un sentido de protección del exterior como del interior. Además, debido a que esta se ha hecho una respuesta crónica ante situaciones que le colocan en sentido de peligro, es a esto a lo que se le llama coraza.

- Transformar positivamente las resistencias mentales hacia las disciplinas que implican el uso del cuerpo. 
- Innovar el diseño curricular mediante comunidades de aprendizaje, trascendiendo la categorización por funcionalidades (adecuaciones), y apostando por la personalización de las actividades en caso de requerirse así con una persona o un grupo específico según sus ritmos de aprendizaje.

- Desarrollar la autodeterminación en las personas, por lo que se entrena la autonomía corporal y la comunicación verbal y no verbal.

- Dar respuesta educativa a necesidades formativas contextualizadas como: integración grupal, desinhibición, conciencia corporal (conocimiento de anatomía y autoimagen, comprensión de la diversidad funcional así como del enfoque en derechos humanos), expresión vocal, autorregulación corporal (relajación, peso, equilibrio, impulsos físicos, acción-reacción), reconocimiento de su propia kinesfera corporal para una mejor orientación espacial), resolución de conflictos (comunicación asertiva/no violenta), estrategias de negociación, herramientas de liderazgo), y finalmente, reconocimiento de la violencia de género, así como mecanismos de autocuidado y de empoderamiento para identificar situaciones con roles de poder.

\section{Conclusiones}

\subsection{El devenir de la operacionalización de la pedagogía teatral desde una perspectiva inclusiva en el CAIPAD APNAE}

El análisis derivado de las distintas limitantes y alcances que conlleva implementar una estrategia socio educativa desde la pedagogía teatral con un enfoque inclusivo, direcciona la mirada hacia las políticas públicas para las personas con diversidad funcional y lo relacionado con la administración educativa del sistema formal, lo que conlleva repensar los canales o medios existentes para los procesos de educación no formal en estos ámbitos, así como las distintas pedagogías alternativas que vienen a fortalecer las necesidades formativas que se sitúan en contextos específicos, como en el APNAE CAIPAD.

A pesar de que la pedagogía teatral tiene diversas dimensiones y campos de acción para los distintos contextos educativos, se ha considerado que la Educación por el Arte, es una dimensión que permite desmitificar las prácticas artísticas con la población con diversidad funcional, personas a las que se les concibe que el abordaje educativo debería ser desde un enfoque terapéutico, lo cual limita el quehacer de otras estrategias y el paradigma en derechos humanos. 
Tal y como lo demuestra el diagnóstico empleado en la metodología de investigación, al ser un campo de análisis la autoimagen y la autonomía corporal, se encuentra que las necesidades formativas apuntan hacia la urgencia de contemplar la alfabetización crítica de forma transversal, la cual no tiene que ver con la lecto-escritura que plantea la educación formal. Debido al rango de edad de las personas que asisten al CAIPAD APNAE y según sus aprendizajes previos (quienes la mayoría no saben leer ni escribir y tampoco hacen uso del lenguaje de señas), las estrategias requieren ir orientadas a fortalecer el lenguaje corporal y vocal, beneficiando indirectamente otras áreas, como las habilidades sociales, por ejemplo. Por lo tanto, es a través del cuerpo que se puede lograr conquistar el área de la comunicación verbal y no verbal, así como de la psicomotricidad, la autorregulación corporal y emocional, la postura, la coordinación, el suavizar los bloqueos psico corporales (corazas), y entre otros temas propios del lenguaje corporal, cuya suma permitirá una movilización física con mayor independencia y calidad de vida. Por ende, el principal hallazgo es que los cuerpos son los principales medios de aprendizaje para transformar posturas de pensamiento, lo que permite generar rupturas ante el capacitismo corporal que critica la Teoría Crip.

Otras necesidades formativas identificadas, desde otras áreas de conocimiento son: comprensión de la violencia de género, la sexualidad en personas con diversidad funcional, la identificación de situaciones de violencia u opresión, y, el reconocimiento de roles de género ( $\mathrm{y}$, por ende, de poder). Los anteriores ejes de la perspectiva de género, vienen a revelar diversas situaciones que han expuesto negativamente a las personas con diversidad funcional en sus cotidianidades, lo que implica que desde otras técnicas y dimensiones de la pedagogía teatral (tal y como lo es el Teatro Aplicado), pueden abordar dichas temáticas de género, posibilitando que lo vivencial produzca aprendizaje significativo, tal y como el enfoque histórico cultural de Vigotsky lo plantea.

Por otra parte, la gestión de procesos educativos inclusivos, así como de la educación corporal desde las artes escénicas, siguen siendo áreas que requieren mayor atención por parte de las instituciones que forman parte de dichos cuerpos institucionales encargados de diseñar e implementar las políticas públicas para el alcance del objetivo de proteger y promover los derechos, la inclusión y la plena participación social de las personas con diversidad funcional, sin embargo, dichas entidades carecen de articulación por lo que los planes, programas y políticas no dan respuesta cuantitativamente hacia un mejoramiento de la accesibilidad hacia la cultura y el arte, por medio de la educación. 
Lo anterior se considera que se debe a la carencia de un departamento encargado de velar por el acceso a la recreación, a la participación en manifestaciones artísticas, así como de complementar sus necesidades formativas con estrategias pertinentes, accesibles con respecto a los distintos niveles educativos, culturales y cognitivos.

Por ende, es necesaria la apertura de un Departamento de Educación Artística, la cual contemple las diversas áreas de conocimiento de las artes y garantice innovación pedagógica para diversos contextos educativos.

Paralelamente, se recomienda que dicho departamento articule con los Departamentos de Educación Especial y el de Apoyos Educativos (MEP) con el fin de que el plan de estudios integre aportes de diversas áreas de conocimiento, pero, sobre todo, recursos que faciliten la inclusión.

Específicamente al Ministerio de Educación Pública, se le recomienda evaluar y replantear su vinculación con la APNAE, para que se lleve a cabo un proceso de organización de forma participativa, con el fin de que se dé apertura al mejoramiento de los estatutos, normativas y plan de estudios. A su vez, resulta fundamental un mapeo de organizaciones, instituciones públicas o privadas, y de personas líderes y lideresas de la comunidad que formen parte de la consolidación de redes de apoyo hacia el CAIPAD (según la ubicación de los distintos centros que existen regionalmente en el país), para que solventen necesidades espaciales y recursos para gastos educativos.

Así, como las aulas integradas de la educación primaria y secundaria tuvieron un proceso administrativo de cambio, para que la niñez pudiera tener acceso a participar de la educación regular a partir del Decreto Ejecutivo 40955 "Establecimiento de la inclusión y la accesibilidad en el Sistema Educativo Costarricense" el cual entró en vigencia en el año 2018. Se considera que es oportuno dar respuesta para las personas adultas y adultas mayores que asisten a los diversos CAIPAD, mejorando la calidad de la educación para quienes asisten como estudiantado, y a su vez beneficiando a los entornos familiares, con el fin de que la transformación surja de manera integral y multidireccional.

Finalmente, la limitación de este estudio, responde a la falta de integración de la población institucional hacia el diagnóstico empleado, como resultado, no se dimensiona la postura de este grupo (personas o entes que tienen relación con los CAIPAD, como: MEP, CONAPDIS y COINDIS), por ende, algunas interrogantes que surgen a partir de dicha limitación son: ¿Cuál es la visión que las instituciones públicas tienen respecto a los CAIPAD? ¿Cuáles son los posicionamientos políticos actuales con respecto a la educación 
para adultos con diversidad funcional? ¿Para qué y cómo se proyectan los procesos formativos para las siguientes generaciones de personas adultas con diversidad funcional? ¿Cuáles políticas públicas se están planteando o se encuentran en proceso de ejecución, que respondan a los derechos culturales y recreativos de las personas adultas con diversidad funcional? ¿Cómo comprende el departamento de carrera docente del MEP la estrategia de pedagogía teatral y de la Administración de la Educación no formal como medios de enseñanza en contextos de educación formal? ¿Qué posibilidades y viabilidades económicas hay de que la misma se incorpore en planes de estudio de este CAIPAD APNAE en específico? Para culminar, se considera fundamental realizar la recomendación de que la estandarización de un currículum educativo para todos los CAIPAD del país no resulta conveniente, debido a que se deben tomar en cuenta las particularidades contextuales para definir las respectivas necesidades formativas de cada centro CAIPAD, las cuales no fueron objeto de estudio en esta investigación por limitaciones en la población de estudio en la metodología planteada, sin embargo, se recomienda valorar las dimensiones de investigación y las categorías de análisis para tomar en cuenta las necesidades formativas para el diagnóstico respectivo en dichos contextos educativos.

\section{Agradecimientos}

1) Al Instituto de Investigación en Educación (INIE) por posibilitar apoyo a los estudiantes de licenciatura mediante becas concursables cada año.

2) A M.Ed. Silvia Camacho, M.Sc. Pamela Jiménez y M.Ed. Melania Monge por acompañarme en mi proceso de investigación.

3) A MET. Paola González por leerme durante la creación de este artículo.

\section{Referencias}

Aguilar, Lorena., Briceño, Gustavo., Valenciano, Ilsie y Chacón, Edgar. (1999). Quien busca...encuentra: elaborando diagnósticos participativos con enfoque de género. San José, Costa Rica: Editorial Unión Mundial para la naturaleza. Recuperado de http://www.colpos.mx/wb/images/Meg/Montecillo/Documentos/mod02.pdf

Alpízar, Marianela., Altonor, Maritza., Alvarado, Joselyn., Alvarado, Margarita., Barrantes, Schirley., Castro, Ericka., ...Solís, Cinthia. (2013). Guía de Orientaciones Técnicas para la implementación del Plan de Estudios de Centros de Atención Integral para Adultos con Discapacidad (CAIPAD). San José, Costa Rica: Editorial Ministerio de Educación Pública de Costa Rica (MEP) y Consejo Nacional de Rehabilitación y Educación Especial (CNREE). 
Álvarez Van der Feltz, Isabel. (2014). La imagen corporal en danza/movimiento terapia. Un estudio con mujeres operadas de cáncer de mama (Tesina de grado de maestría). De la base de datos de la Universidad Autónoma de Barcelona. Depósito Digital de Documentos de la UAB.

Arboleda, Adriana., Medina, Catalina., Vergara, Felipe., Kleutgens, Inge y Cancino, Nicolás. (2011). Teatro para la transformación social. Herramientas pedagógicas. Tomo 3. Recuperado de https://www.ziviler-friedensdienst.org/sites/zivilerfriedensdienst.org/files/anhang/publikation/zfd-teatro-para-la-transformacion-social101240.pdf

Asociación Pro Niño y Adulto Excepcional. (2011). Reglamento para el manejo conductual interno Asociación Pro Niño y Adulto Excepcional. Heredia, Costa Rica: APNAE CAIPAD.

Astrosky, Débora. (21 de setiembre de 2015). Pedagogía Teatral, una mirada posible I. [Grupo de discusión de seminario virtual]. Recuperado de https://www.celcit.org.ar/cursos-y-talleres/21/cursos-a-distancia/33/taller-pedagogiateatral-una-mirada-posible-1-clases-estudiar/

Bairábar, José., Lamata, Rafael., Domínguez, Rosa., Bonell, Lars., Casellas, Lorenzo. y Gamonal, Antonio. (2003). La construcción de procesos formativos en educación no formal. Madrid, España: Ediciones Narcea S.A.

Benavides, Zaida. (s.f). Arte y creatividad en el niño o niña con discapacidad. San José, Costa Rica: Editorama S.A.

Blanco, Rosa. (2002). La educación inclusiva en América Latina realidades y perspectivas. San José, Costa Rica: Centro Nacional de Recursos para la Inclusión Educativa.

Brugarolas, María Luisa. (2015). El cuerpo plural. Danza integrada a la inclusión. Una renovación de la mirada (Tesis doctoral no publicada). De la base de datos de Universitat Politècnica de València. doi:10.4995/Thesis/10251/62203.

Castillo, Rocío. (2011). Hacia una nueva espiritualidad desde la pedagogía holística. Revista Ensayos Pedagógicos, 6(2), 55-70. Recuperado de https://www.revistas.una.ac.cr/index.php/ensayospedagogicos/article/view/5819/5687

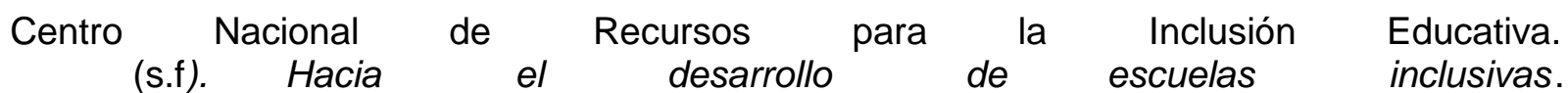
Recuperado de http://www.inclusioneducativa.org/content/documents/MARCO GENERAL DE LA El . pdf

Chaves, Ana. (2001). Implicaciones educativas de la teoría sociocultural de Vygotsky. Revista Educación, 25(2), 59-65. Recuperado de http://www.redalyc.org/articulo.oa?id=44025206 
Collado, Adriana. (s.f). La construcción conjunta de políticas de educación artística entre el Ministerio de Educación Pública y el Ministerio de Cultura, Juventud y Deportes de Costa Rica. Recuperado de www.lacult.unesco.org/docc/PonenciaCostaRica.pdf

Convención sobre los derechos de las personas con discapacidad. Compendio Ley 8661. (2009). San José, Costa Rica: Consejo Nacional de Rehabilitación y Educación Especial.

De León, A. (s.f). Psicología del cuerpo Wilhelm Reich. Recuperado de http://www.psicont.com/ftp/reich-psicocuerpo.pdf

Fernández, Daniel. (2016). Educación de Personas con diversidad Funcional. Algunos apuntes históricos. Actualidades investigativas en educación. 16(3). 1-23. Doi 10.15517/AIE.V16I3.26064

García, Verónica. (1996). Manual de Pedagogía Teatral. Recuperado de http://www.memoriachilena.cl/archivos2/pdfs/MC0050907.pdf

Hacia el desarrollo de escuelas inclusivas. (s.f). Educación Inclusiva. Recuperado de http://www.inclusioneducativa.org/content/documents/MARCO GENERAL DE LA El .pdf

Hidalgo, Rocío. (2015). Habitando cuerpos habitados, imaginarios y proceso creativo. Iztapalapa, México: Ediciones del Lirio S.A de C.V.

Instituto Nacional de Estadística y Censos. (2018). Encuesta Nacional sobre la discapacidad en Costa Rica. Recuperado de http://www.inec.go.cr/multimedia/enadis-2018-ladiscapacidad-en-costa-rica

Ley 7600 lgualdad de oportunidades para las personas con discapacidad. (2015). San José, Costa Rica: Consejo Nacional de Rehabilitación y Educación Especial.

Maya, Arnobio. (2013). ¡Urgente! ¡La educación debe de cambiar! Transitando desde la educación tradicional a la educación holística. San José, Costa Rica: Editorial Coordinación Educativa y Cultural Centroamericana (CECC/SICA).

Meléndez, Raudin. (2019). Las políticas públicas en materia de discapacidad en América Latina y su garantía de acceso a una educación inclusiva de calidad, Actualidades Investigativas en Educación, 19(2), 1-26. doi: 10.15517/AIE.V19I2.36916

Organización de las Naciones Unidas para la Educación, la Ciencia y la Cultura (Unesco). (2000. Educación para Todos: cumplir nuestros compromisos comunes. Foro Mundial sobre la Educación Marco de Acción de Dakar.

Santos, Miguel. (2000). El pensamiento complejo y la pedagogía. Bases para una teoría holística de la educación, Estudios Pedagógicos 26, 133-148. Recuperado de http://www.redalyc.org/articulo.oa?id=173514139012 
Shalock, Robert. (1999). Hacia una nueva concepción de la discapacidad. Recuperado de http://campus.usal.es/ inico/investigacion/jornadas/jornada3/actas/conf6.pdf

Varela, Luis. (s.f). Inclusión excluyente en la educación. Recuperado de http://observatorio.inie.ucr.ac.cr/hablemos-de-educacion/30-inclusion-excluyenteeducacion

Varela, Luis. (2015). Matriz curricular excluyente y adecuaciones curriculares en el actual proyecto educativo costarricense. Recuperado de http://observatorio.inie.ucr.ac.cr/attachments/article/38/Curriculum\%20estandarizado. $\underline{\mathrm{pdf}}$ 
Revista indizada en

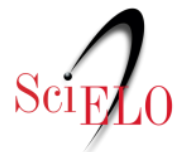

redalyc satindex

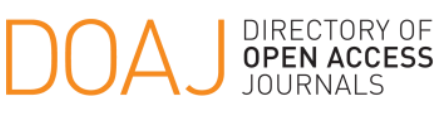

Distribuida en las bases de datos:

- Dialnet

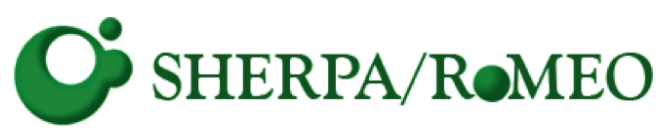

REDIB

Red Iberoamericana

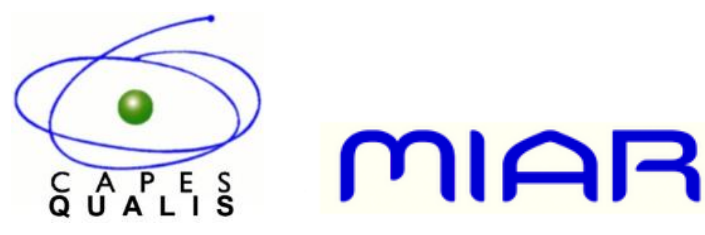

